\title{
The neglected Plasmodium vivax: are researchers from endemic areas really concerned about new treatment options?
}

\author{
O Plasmodium vivax negligenciado: os pesquisadores de áreas \\ endêmicas estão mesmo preocupados \\ com novas opções de tratamento?
}

\section{Dear Editor:}

The global burden of malaria caused by Plasmodium vivax is approximately 80 million cases annually. Outside Africa, Plasmodium vivax accounts for more than $50 \%$ of all malaria cases, with the majority (80-90\%) occurring in the Middle East, Asia and the Western Pacific, and $10-20 \%$ in Central and South America $^{7}$. It is usually considered to be a benign disease; however, there are reports of increasing clinical severity ${ }^{56}$ and resistance to antimalarials ${ }^{128}$ in South America and Southeast Asia. If mortality due to malaria is not an issue of priority in Plasmodium vivax endemic areas, then attention should be given to the absenteeism from work and school and the anemia that this disease produces, which certainly impairs the sustained development of these areas ${ }^{11}$. The highly significant economic impact of Plasmodium vivax malaria mandates that more resources be directed specifically to research on this parasite, including studies on pathogenesis and drug resistance.
Figure 1 shows the number of controlled, randomized clinical trials with antimalarials for Plasmodium falciparum or Plasmodium vivax on the respective continent where the study was conducted, from January 1996 to December 2005, identified through MEDLINE (key words searched in the MeSH database: malaria, vivax; Plasmodium vivax; malaria, falciparum; Plasmodium falciparum; therapeutics; therapy; treatment outcome; treatment failure; clinical protocols; therapies, investigational). Only 31 studies were performed with antimalarials for Plasmodium vivax over this 10-year period. Surprisingly, most of the articles published in Asia and the Americas, where Plasmodium vivax is more common than Plasmodium falciparum, relate to antimalarials for treating Plasmodium falciparum.

Chloroquine is still the drug of choice for treating Plasmodium vivax malaria around the world. The increasing resistance to this drug has not yet led to any studies on the schizonticidal drugs that are used routinely for Plasmodium falciparum, as

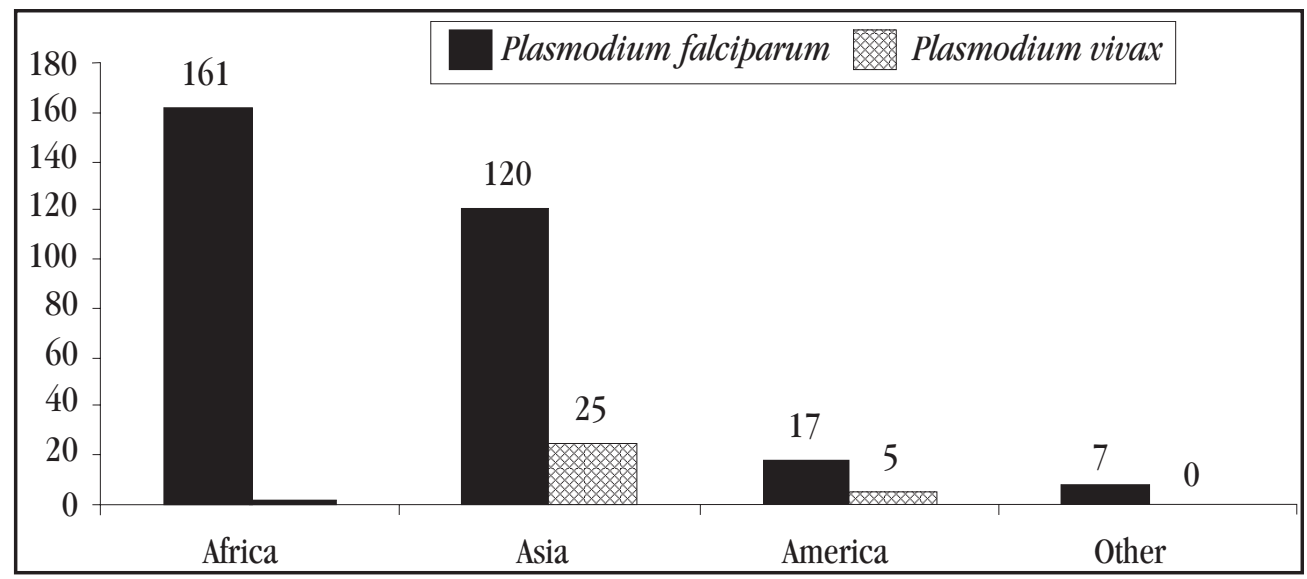

Figure 1 - Number of published controlled randomized clinical trials on antimalarials for Plasmodium falciparum and Plasmodium vivax, from 1996 to 2005 (MEDLINE), according to the continent on which the study was conducted.

\footnotetext{
1. Gerência de Malária, Fundação de Medicina Tropical do Amazonas, Manaus, AM. 2. Universidade do Estado do Amazonas, Manaus, AM. 3. Centro Universitário Nilton Lins, Manaus, AM. 4. Drugs for Neglected Diseases initiative (DND $i$ ), Latin American Liaison, Rio de Janeiro, RJ.

Address to: Dr. Marcus Vinícius Guimarães de Lacerda. Gerência de Malária da FMT-AM. Av. Pedro Teixeira 25, 69040-000 Manaus, AM, Brasil.

Telefax: 559236560620

e-mail: marcuslacerda@uol.com.br

Recebido em: 18/12/2006

Aceito em: 11/07/2007
} 
potential alternatives for Plasmodium vivax. Chloroquine was developed in 1934, is very cheap (US\$ 0.10 per treatment) and easy to administer, and has few side effects. Nonetheless, new drugs and therapeutic schemes are needed to treat the growing number of patients who cannot be cured with chloroquine? Artemisinin-based combination therapy (ACT) has been strongly recommended by the World Health Organization for treating Plasmodium falciparum. Systematic testing against Plasmodium vivax should also be considered in order to simplify treatment schemes and/or evaluate ACT as an alternative when resistance emerges and becomes widespread.

Artesunate plus mefloquine is a highly effective combination against resistant Plasmodium falciparum ${ }^{4}$ and is under field investigation in Brazil. Artemether plus lumefantrine has already been adopted as the first-line option for non-severe falciparum malaria treatment in some Brazilian endemic areas since 2006. These ACTs may provide not only an effective alternative for treating this often lethal parasite, but should also be considered highly relevant in relation to the emergence of Plasmodium vivax, among countries that are committed to decreasing malaria morbidity in the Americas.

Radical cure for Plasmodium vivax malaria (by killing the hypnozoites) is achieved only with primaquine. In cases of resistance to this drug or when contraindications such as G6PD deficiency exist, no other drug is available. Tafenoquine is a promising new 8-aminoquinoline that still needs additional Phase III clinical trials ${ }^{10}$ and regulatory approval. The lack of a good in vitro protocol for Plasmodium vivax cultivation, which may also unfortunately be regarded as having been a non-priority issue in recent years, immensely restricts the preclinical development of additional new drugs against this parasite.

The declining efficacy of current therapies for Plasmodium vivax highlights the need for new treatment options. Underestimating the impact of Plasmodium vivax on the global health system may dramatically impair the achievement of one of the 2015 United Nations Millennium Development Goals, i.e. a commitment to have halted and begun to reverse the incidence of malaria and other major diseases by that date (http://www. un.org/millenniumgoals/).

Malaria, as a single entity, is recognized as a prominent neglected disease, without much research and development investment devoted to it. However, most of the activity is perhaps justifiably focused on Plasmodium falciparum, because of its more lethal consequences. Nevertheless, the important health and economic morbidity that Plasmodium vivax imposes upon a much larger number of people in certain areas requires committed support for baseline mapping of its prevalence, new scientifically proven treatment protocols, and appropriate alignment of research efforts with regional and national health policies ${ }^{3}$.

In our view, the serious risk posed by Plasmodium vivax malaria in endemic areas is not fully recognized and acknowledged. Only 31 Plasmodium vivax drug studies reported over a 10-year period illustrates the poor level of interest in this vital area. This particular malarial species may become a much more prominent neglected disease in the immediate future if specific Plasmodium vivax research is not greatly enhanced.

\section{REFERENCES}

1. Alecrim MGC, Alecrim W, Macedo V. Plasmodium vivax resistance to chloroquine (R2) and mefloquine (R3) in Brazilian Amazon region. Revista da Sociedade Brasileira de Medicina Tropical 32:67-68, 1999.

2. Canessa A, Mazzarello G, Cruciani M, Bassetti D. Chloroquine-resistant Plasmodium vivax in Brazil. Transactions of the Royal Society of Tropical Medicine and Hygiene 86:570-571, 1992.

3. Hotez PJ, Molyneux DH, Fenwick A, Ottesen E, Sachs SE, Sachs JD. Incorporating a rapid-impact package for neglected tropical diseases with programs for HIV/AIDS, Tuberculosis, and Malaria. PLoS. Medicine 3:e102, 2006.

4. Hutagalung R, Paiphun L, Ashley E, McGready R, Brockman A, Thwai K, Singhasivanon P, Jelinek T, White N, Nosten F. A randomized trial of artemetherlumefantrine versus mefloquine-artesunate for the treatment of uncomplicated multi-drug resistant Plasmodium falciparum on the western border of Thailand. Malaria Journal 4:46, 2005.

5. Kochar DK, Saxena V, Singh N, Kochar SK, Kumar SV, Das A. Plasmodium vivax malaria. Emerging Infectious Diseases 11:132-134, 2005.

6. Lomar AV, Vidal JE, Lomar FP, Barbas CV, Matos GJ, Boulos M. Acute respiratory distress syndrome due to vivax malaria: case report and literature review. Brazilian Journal of Infectious Diseases 9:425-430, 2005.

7. Mendis K, Sina BJ, Marchesini P, Carter R. The neglected burden of Plasmodium vivax malaria. American Journal of Tropical Medicine and Hygiene 64: 97-106, 2001.

8. Sumawinata IW, Bernadeta, Leksana B, Sutamihardja A, Purnomo, Subianto B, Sekartuti, Fryauff DJ, Baird JK. Very high risk of therapeutic failure with chloroquine for uncomplicated Plasmodium falciparum and P. vivax malaria in Indonesian Papua. American Journal of Tropical Medicine and Hygiene 68:416-420, 2003.

9. Vinetz JM. Emerging chloroquine-resistant Plasmodium vivax (Benign Tertian) malaria: the need for alternative drug treatment. Clinical Infectious Diseases 42:1073-1074, 2006.

10. Walsh DS, Wilairatana P, Tang DB, Heppner DG, Brewer TG, Krudsood S, Silachamroon U, Phumratanaprapin W, Siriyanonda D, Looareesuwan S. Randomized trial of 3-dose regimens of tafenoquine (WR238605) versus lowdose primaquine for preventing Plasmodium vivax malaria relapse. Clinical Infectious Diseases 39:1095-1103, 2004.

11. Zamora F, Ramirez 0, Vergara J, Arévalo-Herrera M, Herrera S. Hemoglobin levels related to days of illness, race, and Plasmodium species in Colombian patients with uncomplicated malaria. American Journal of Tropical Medicine and Hygiene 73:50-54, 2005.

\section{Marcus Vinícus Guimarães de Lacerda ${ }^{1,2,3}$, Christina Zackiewicz ${ }^{4}$, Wilson Duarte Alecrim ${ }^{1}$ and Maria das Graças C. Alecrim ${ }^{2,3}$}

